CERN-TH.6627/92

UICHEP-TH/92-13

hep-th/9209006

\title{
R-Matrix Formulation of KP Hierarchies and Their Gauge Equivalence
}

\author{
H. Aratyn円 \\ Department of Physics , University of Illinois at Chicago \\ Box 4348, Chicago, Illinois 60680, U.S.A. , e-mail : u23325@uicvm \\ E. Nissimov ${ }^{2}$ and S. Pacheva ${ }^{2}$ \\ Department of Physics, Ben-Gurion University of the Negev \\ Box 653, IL-84105 Beer Sheva, Israel \\ e-mail : emil@bguvms, svetlana@bguvms \\ and \\ Theory Division, CERN, CH-1211 Geneva 23, Switzerland \\ e-mail : nissimov@cernvm, svetlana@vxcern \\ I. Vaysburd \\ Racah Institute of Physics, Hebrew University \\ IL-91904 Jerusalem, Israel, e-mail : igor@hujivms
}

\begin{abstract}
The Adler-Kostant-Symes $R$-bracket scheme is applied to the algebra of pseudodifferential operators to relate the three integrable hierarchies: KP and its two modifications, known as nonstandard integrable models. All three hierarchies are shown to be equivalent and connection is established in the form of a symplectic gauge transformation. This construction results in a new representation of the W-infinity algebras in terms of 4 bosonic fields.
\end{abstract}

CERN-TH.6627/92

August 1992

\footnotetext{
${ }^{1}$ Work supported in part by U.S. Department of Energy, contract DE-FG02-84ER40173

${ }^{2}$ On leave from : Institute of Nuclear Research and Nuclear Energy, Boul. Trakia 72, BG-1784 Sofia, Bulgaria.
} 


\section{Introduction}

One of the important and still unsolved problems of the two-dimensional physics is to describe consistently systems with infinitely many functional (field-) degrees of freedom. Among such systems the largest attention was attained by the Kadomtsev-Petviashvili $(K P)$ completely integrable hierarchy which proved to be relevant for a variety of the physical problems. A recent and intriguing development in this field is the appearance of the integrable hierarchies, including $K P$, in the matrix models known to describe at multicritical points $c \leq 1$ matter systems coupled to $D=2$ quantum gravity [1]. In particular, the partition function of both discrete matrix models [2, 3] and of continuum $c \leq 1$ string field theory [4, 5] is expressed in terms of a constrained $\tau$-function of the $K P$ hierarchy. The coupling constants in the matrix model partition function, corresponding to various possible deviations from the critical points, are the evolution parameters in the $K P$ hierarchy.

The most essential feature of the integrable hierarchies with infinite number of degrees of freedom, which prompts their connection to $2 D$ conformal field theories, $c \leq 1$ strings and their matrix model counterparts, is their Hamiltonian structure [6, 7]. Thus, the centerless Virasoro algebra provides the second Hamiltonian structure of Korteweg-de-Vries (KdV) hierarchy. This is the algebra of constraints on the partition function of the one-matrix model [1]. In the same way $W_{N}$ algebras [8], or more precisely, their semiclassical analogs the Gelfand-Dickey algebras [9], give the second Hamiltonian structures of the generalized KdV hierarchies. $W_{N}$ are the algebras of constraints on the partition function in multi-matrix and Kontsevich matrix models [2, 3]. Finally, $\mathbf{W}_{\mathbf{1}+\infty}$ algebra [10], which is isomorphic to the Lie algebra of differential operators on the circle $\mathcal{D O P}\left(S^{1}\right)$ [11], yields the first Hamiltonian structure of $K P$ hierarchy [12, 13, 14].

An important open problem of the matrix model formulation of $2 D$ quantum gravity is how to describe the interpolation between two different vacua (one, characterized by $(p, q)$ conformal matter and another one - by $\left.\left(p^{\prime}, q^{\prime}\right), p^{\prime} \neq p, q^{\prime} \neq q\right)$. It is known [15] that this cannot be solved in terms of the ordinary $K P$ evolution parameters. A hope to solve this problem is to analyze all integrable hierarchies which are equivalent to $K P$. This is also an interesting mathematical problem by its own value.

In this letter we would like to contribute to this program. The powerful Adler-KostantSymes (AKS) scheme for Lie-algebraic construction of integrable models (substantially improved and extended by Reyman and Semenov-Tian-Shansky) [16 is applied to the algebra of pseudo-differential operators on the circle $\Psi \mathcal{D O}\left(S^{1}\right)$ [17]. This scheme permits the treatment of the three different integrable $K P$-like hierarchies - ordinary $K P$ and its two modifications , known as nonstandard integrable models [13], on equal footing. They correspond to the three possible splittings of the algebra $\Psi \mathcal{D O}\left(S^{1}\right)$ into a linear sum of two subalgebras. The main result is that all three hierarchies are proved to be "gauge" equivalent via a generalized Miura transformation. The (field-dependent) "gauge" transformations, which are explicitly constructed, belong to well-defined subgroups of the formal group of pseudo-differential operators - the abelian group of operators of multiplication by a function and the group of diffeomorphisms on the circle (Virasoro group), respectively. For the first of the modified $K P$ hierarchies, this "gauge" equivalence was previously established [18] in the "semiclassical" limit of $K P$ (known in nonlinear hydrodynamics as Benney integrable hierarchy [19]). 
As an important byproduct, the above "gauge" transformations together with the AKS $R$-bracket scheme provide new explicit realizations of $\mathbf{W}_{\mathbf{1}+\infty}$ algebras in terms of an unconventional set of four Bose fields.

\section{The Adler-Kostant-Symes Scheme and Applications to KP Hi- erarchies}

\subsection{General Scheme and R Operators}

It is well-known that the Lie algebra methods allow for an unifying treatment of integrable systems [6]. One of the main purpose of this paper is to describe a relation between the Lax formulation of various $K P$-type systems defined below and the $R$-operator approach [20, 21] to the integrable systems based on the AKS scheme [16]. We first recall the notion of integrability.

Complete Integrability: Consider a Hamiltonian system with $n$ degrees of freedom possessing standard Hamiltonian structure with Hamiltonian $H(p, q)$ and Poisson bracket $\{\cdot, \cdot\}$. A Hamiltonian system is called completely (or Liouville) integrable if it has $n$ conserved quantities (integrals of motion) $I_{k}(p, q), k=1, \ldots n$, which are in involution: $\left\{I_{i}, I_{j}\right\}=0$. For such a system we can find the action-angle variables and write the general solution to the equations of motion.

Lax formulation: For infinite-dimensional integrable Hamiltonian systems there exists the convenient Lax (or "zero-curvature") formulation [6]. In the Lax formulation the dynamical equations of motion can be written in terms a Lax pair $L, P$, with values in some Lie algebra $\mathcal{G}$, as the Lax-type equation

$$
\frac{d L}{d t}=[L, P]
$$

The Lax formulation leads straightforwardly to construction of the integrals of motion. Namely, for any Ad-invariant function $I$ on $\mathcal{G}, I(L)$ is a constant of motion. In fact, it can be shown that any completely integrable Hamiltonian system admits a Lax representation (at least locally) [22].

The KP hierarchy: An important example of integrable system admitting the Lax formulation is given by $K P$ hierarchy consisting of the following family of Lax equations

$$
\frac{\partial L}{\partial t_{r}}=\left[L, L_{+}^{r}\right], \quad r=1,2,3, \ldots
$$

where $L$ is a pseudo-differential operator

$$
L=D+\sum_{i=1}^{\infty} u_{i} D^{-i}
$$

The subscript $(+)$ means taking the purely differential part of $L^{r}$ and $t=\left\{t_{r}\right\}$ are the evolution parameters (infinitely many time coordinates). The flows (2) are bi-Hamiltonian 
[7], i.e. there exist two Poisson bracket structures $\{\cdot, \cdot\}_{1,2}$, such that we can rewrite (2) as :

$$
\frac{\partial L}{\partial t_{r}}=\left\{H_{r}, L\right\}_{2}=\left\{H_{r+1}, L\right\}_{1}
$$

Here the Hamiltonians for the $K P$ hierarchy are $H_{r}=\frac{1}{r} \int \operatorname{Res} L^{r}$ ( Res denotes the coefficient in front of $D^{-1}$ ). The second equality in (4) is a particular case of the so called Lenard relations $\left\{H_{r+1}, L\right\}_{m}=\left\{H_{r}, L\right\}_{m+1}$ for a hierarchy of Poisson bracket structures $m=$ $1,2, \ldots$ There exists a fundamental theorem (see e.g. [23]) connecting notion of integrability with the property of possessing a bi-Hamiltonian structure, which establishes the $K P$ system as integrable.

The AKS Scheme: A very wide class of integrable models can be constructed through the application of the AKS method having roots in the coadjoint orbit formulation.

Let $G$ denote a Lie group and $\mathcal{G}$ be its Lie algebra. $G$ acts on $\mathcal{G}$ by the adjoint action: $A d(g) X=g X g^{-1}$ with $g \in G$ and $X \in \mathcal{G}$. Let $\mathcal{G}^{*}$ be the dual space of $\mathcal{G}$ relative to a non-degenerate bilinear form $\langle\cdot \mid \cdot\rangle$ on $\mathcal{G}^{*} \times \mathcal{G}$. The corresponding coadjoint action of $G$ on $\mathcal{G}^{*}$ is obtained from duality of $\langle\cdot \mid \cdot\rangle:\left\langle A d^{*}(g) U \mid X\right\rangle=\left\langle U \mid A d\left(g^{-1}\right) X\right\rangle$. We will denote the infinitesimal versions of adjoint and coadjoint transformations by $\operatorname{ad}(Y)$ and $a d^{*}(Y)$ (for $g=\exp Y)$.

There exists a natural Poisson structure on the space $C^{\infty}\left(\mathcal{G}^{*}, \mathbb{R}\right)$ of smooth, real-valued functions on $\mathcal{G}^{*}$ called Lie-Poisson $(\mathrm{LP})$ bracket. The LP bracket for $F, H \in C^{\infty}\left(\mathcal{G}^{*}, \mathbb{R}\right)$ is given by :

$$
\{F, H\}(U)=-\langle U \mid[\nabla F(U), \nabla H(U)]\rangle
$$

where the gradient $\nabla F: \mathcal{G}^{*} \rightarrow \mathcal{G}$ is defined by the standard formula $\left.\frac{d}{d t} F(U+t V)\right|_{t=0}=$ $\langle V \mid \nabla F(U)\rangle$ and where $[\cdot, \cdot]$ is the standard Lie bracket on $\mathcal{G}$. It follows clearly that $\{\cdot, \cdot\}$ is antisymmetric and it is also easy to verify the Jacobi identity. On each orbit in $\mathcal{G}^{*}$ the LP bracket gives rise to a non-degenerate symplectic structure. Moreover, for any Hamiltonian $H$ on such orbit we have a Hamiltonian equation $d U / d t=a d^{*}(\nabla H(U)) U$.

We now introduce $R$-operator (generalized $R$-matrix) as a linear map from a Lie algebra $\mathcal{G}$ to itself such that the bracket :

$$
[X, Y]_{R} \equiv \frac{1}{2}[R X, Y]+\frac{1}{2}[X, R Y]
$$

defines a second Lie structure on $\mathcal{G}$ [20]. The modified Yang-Baxter equation (YBE) for the $R$-matrix must hold in order to ensure the Jacobi relation.

We can furthermore introduce a new LP bracket $\{\cdot, \cdot\}_{R}$ called $R$-bracket substituting the usual Lie bracket $[\cdot, \cdot]$ by the $R$-Lie bracket $[\cdot, \cdot]_{R}(6)$ in (5):

$$
\{F, H\}_{R}(U)=-\left\langle U \mid[\nabla F(U), \nabla H(U)]_{R}\right\rangle
$$

A function $H$ on $\mathcal{G}^{*}$ is called $A d^{*}$-invariant (Casimir) if $H\left[A d^{*}(g) U\right]=H[U]$ or, infinitesimally, $a d^{*}(\nabla H(U))(U)=0$ for each $U \in \mathcal{G}^{*}$. Then one can show [20] that : 
(1) the $a d^{*}$-invariant functions are in involution with respect to both brackets (5) and (7) ;

(2) the Hamiltonian equation on $\mathcal{G}^{*}$ takes the following (generalized Lax) form :

$$
d U / d t=\frac{1}{2} a d^{*}(R(\nabla H(U))) U
$$

corresponding to the equations of motion $d F / d t=\{H, F\}_{R}$ for $F \in C^{\infty}\left(\mathcal{G}^{*}, \mathbb{R}\right)$.

Hence the above R-matrix technique leads to a direct construction of integrable systems based on Casimir functions on $\mathcal{G}^{*}$. The basic realization of this technique arises when the Lie algebra $\mathcal{G}$ decomposes as a vector space into two subalgebras $\mathcal{G}_{+}$and $\mathcal{G}_{-}$i.e. $\mathcal{G}=\mathcal{G}_{+} \oplus \mathcal{G}_{-}$. Let $P_{ \pm}$be the corresponding projections on $\mathcal{G}_{ \pm}$. Then $R=P_{+}-P_{-}$satisfies the modified YBE and provides a specific realization for the above scheme.

\subsection{AKS Construction of Three KP Hierarchies}

Here we will illustrate the AKS construction on $\mathcal{G}=\Psi \mathcal{D O}\left(S^{1}\right)$ - the Lie algebra of pseudo-differential operators on circle. Recall that arbitrary pseudo-differential operator $X\left(x, D_{x}\right)=\sum_{k \geq-\infty} X_{k}(x) D_{x}^{k}$ is conveniently represented by its symbol [17] - a Laurent series in the variable $\xi$ :

$$
X(\xi, x)=\sum_{k \geq-\infty} X_{k}(x) \xi^{k}
$$

and the operator multiplication corresponds to the following symbol multiplication :

$$
X(\xi, x) \circ Y(\xi, x)=\sum_{N \geq 0} h^{N} \frac{1}{N !} \frac{\partial^{N} X}{\partial \xi^{N}} \frac{\partial^{N} Y}{\partial x^{N}}
$$

which determines a Lie algebra structure given by a commutator $[X, Y] \equiv \frac{1}{h}(X \circ Y-Y \circ X)$. Explicitly we have :

$$
[X(\xi, x), Y(\xi, x)]=\sum_{N \geq 1}(h)^{N-1} \frac{1}{N !}\left(\frac{\partial^{N} X}{\partial \xi^{N}} \frac{\partial^{N} Y}{\partial x^{N}}-\frac{\partial^{N} X}{\partial x^{N}} \frac{\partial^{N} Y}{\partial \xi^{N}}\right)
$$

The constant $h$ appearing in (10) and (11) has the meaning of a deformation parameter and henceforth will be taken $h=1$. The limit $h \rightarrow 0$ defines the semiclassical limit of $\Psi \mathcal{D O}\left(S^{1}\right)$ where the Lie bracket (111) reduces to a two-dimensional Poisson bracket: $[X(\xi, x), Y(\xi, x)]=$ $(\partial X / \partial \xi)(\partial Y / \partial x)-(\partial X / \partial x)(\partial Y / \partial \xi)$.

Using the Adler trace one next defines an invariant, non-degenerate bilinear form :

$$
\langle L \mid X\rangle \equiv \operatorname{Tr}_{A}(L X)=\int d x \operatorname{Res}_{\xi} L(\xi, x) \circ X(\xi, x)
$$

which allows an identification of the dual space $\mathcal{G}^{*}$ with $\mathcal{G}$ and the coadjoint action with the adjoint action.

There exist three natural decompositions of $\mathcal{G}$ into a linear sum of two subalgebras:

$$
\mathcal{G}_{+}^{\ell}=\left\{X_{+} \equiv X_{\geq \ell}=\sum_{i=\ell}^{\infty} X_{i}(x) \xi^{i}\right\} \quad ; \quad \mathcal{G}_{-}^{\ell}=\left\{X_{-} \equiv X_{<\ell}=\sum_{i=-\ell+1}^{\infty} X_{-i}(x) \xi^{-i}\right\}
$$


labeled by the index $\ell$ taking three values $\ell=0,1,2$. For each $\ell$ we clearly have $\mathcal{G}=\mathcal{G}_{+}^{\ell} \oplus \mathcal{G}_{-}^{\ell}$. Correspondingly the dual spaces to subalgebras $\mathcal{G}_{ \pm}^{\ell}$ are given by :

$$
\mathcal{G}_{+}^{\ell *}=\left\{L_{-} \equiv L_{<-\ell}=\sum_{i=\ell+1}^{\infty} \xi^{-i} \circ u_{-i}(x)\right\} \quad ; \quad \mathcal{G}_{-}^{\ell^{*}}=\left\{L_{+} \equiv L_{\geq-\ell}=\sum_{i=-\ell}^{\infty} \xi^{i} \circ u_{i}(x)\right\}
$$

Note that in (14) the differential operators are put to the left.

Denoting by $R_{\ell}=P_{+}-P_{-}$for each of three cases one finds that $R$-bracket is given by :

$$
[X, Y]_{R_{\ell}}=\left[X_{\geq \ell}, Y_{\geq \ell}\right]-\left[X_{<\ell}, Y_{<\ell}\right]
$$

Furthermore, from the general relation for the $R$-coadjoint action of $\mathcal{G}$ on its dual space $a d_{R}^{*}(X) L=\frac{1}{2} a d^{*}(R X) L+\frac{1}{2} R^{*} a d^{*}(X) L$ we find the that the infinitesimal shift along an $R$-coadjoint orbit $\mathcal{O}\left(R_{\ell}\right)$ has the form :

$$
\delta_{R_{\ell}} L \equiv a d_{R_{\ell}}^{*}(X) L=\left(a d^{*}\left(X_{+}\right) L_{-}\right)_{-}-\left(a d^{*}\left(X_{-}\right) L_{+}\right)_{+} \equiv\left[X_{\geq \ell}, L_{<-\ell}\right]_{<-\ell}-\left[X_{<\ell}, L_{\geq-\ell}\right]_{\geq-\ell}
$$

Henceforth, the subscripts \pm will denote projections on $\mathcal{G}_{\ell \pm}$ and $\mathcal{G}_{\ell \mp}^{*}$, as in (13), (14). Also, we shall skip the sign $\circ$ in symbol products for brevity.

We will now discuss in greater detail the Hamiltonian structure of the integrable systems given by the three decompositions labeled by $\ell=0,1,2$ as defined by the AKS scheme with Hamiltonian equations of motion (8). We will call the resulting hierarchies the $r-K P$ or $\mathbf{K} \mathbf{P}_{\ell}$ hierarchies.

$\mathbf{K P}_{\ell=\mathbf{0}}$ : Here we take $R$-coadjoint orbit of the form $\mathcal{O}\left(R_{0}\right)=\left\{L=\xi+L_{-} \equiv \xi+\right.$ $\left.\sum_{k=1}^{\infty} \xi^{-k} u_{k}(x)\right\}$. Choosing as a Casimir function $H_{m+1}=\frac{1}{m+1} \int d x \operatorname{Res}_{\xi} L^{m+1}$ we get from (8):

$$
\frac{\partial L}{\partial t_{m}}=\frac{1}{2} a d^{*}\left(\left(\nabla H_{m+1}\right)_{+}-\left(\nabla H_{m+1}\right)_{-}\right) L=a d^{*}\left(\left(L^{m}\right)_{+}\right) L
$$

with $\left(L^{m}\right)_{+}=\sum_{j \geq 0}\left(\delta H_{m+1} / \delta u_{j+1}(x)\right) \xi^{j}$. We recognize in (17) the standard $K P$ flow equation (21). One finds the corresponding Hamiltonian structure to be induced by the LP structure: $\left\{u_{i}(x), u_{j}(y)\right\}_{R_{0}}=\Omega_{i-1, j-1}^{(\ell=0)}(u(x)) \delta(x-y)$ where the form on the right hand side is given by [12, 13]:

$$
\Omega_{i, j}^{(\ell)}(u(x))=-\sum_{k=0}^{i+\ell}\left(\begin{array}{c}
i+\ell \\
k
\end{array}\right) u_{i+j+\ell-k+1}(x) D_{x}^{k}+\sum_{k=0}^{j+\ell}(-1)^{k}\left(\begin{array}{c}
j+\ell \\
k
\end{array}\right) D_{x}^{k} u_{i+j+\ell-k+1}(x)
$$

for $\ell=0$. This LP bracket algebra is isomorphic to the centerless $\mathbf{W}_{\mathbf{1}+\infty}$ algebra 14. In conclusion we have found that $\mathbf{K P}_{\ell=\mathbf{0}}$ is the standard $K P$ hierarchy.

$\mathbf{K P}_{\ell=\mathbf{1}}$ : Here we first consider elements of $\mathcal{G}_{-}^{1}{ }^{*}$ of the type $L_{+}=\xi+u_{0}+\xi^{-1} u_{1}$, which preserve their form under $\delta_{R_{1}} L_{+}=a d_{R_{1}}^{*}(X) L_{+}$, i.e. they span an $R_{1}$-orbit of finite functional dimension 2 . Calculation of the Poisson bracket according to (7) :

$$
\left\{\left\langle L_{+} \mid X\right\rangle,\left\langle L_{+} \mid Y\right\rangle\right\}_{R_{1}}=-\left\langle L_{+} \mid[X, Y]_{R_{1}}\right\rangle
$$

yields the $R$-brackets: $\left\{u_{0}(x), u_{1}(y)\right\}_{R_{1}}=-\delta^{\prime}(x-y)$ and zero otherwise. We then define a complete Lax operator defined as $L^{(1)}=L_{+}+L_{-}=\xi+u_{0}+\xi^{-1} u_{1}+\sum_{i \geq 2} \xi^{-i} v_{i-2}$. 
Application of (19) gives a Hamiltonian structure being a direct sum of the matrix $P^{(1)}$ associated to the modes $\left\{u_{0}, u_{1}\right\}$ and the Hamiltonian structure $\Omega^{(1)}$ associated to $\left\{v_{i} \mid i \geq 0\right\}$ [13] :

$$
\left(\begin{array}{cc}
P^{(1)} & 0 \\
0 & \Omega^{(1)}
\end{array}\right) \text { with } P^{(1)}=\left(\begin{array}{cc}
0 & -\partial \\
-\partial & 0
\end{array}\right)
$$

Note that $\Omega^{(1)}$ (18) corresponds to the centerless $\mathbf{W}_{\infty}$ algebra.

$\mathbf{K P}_{\ell=\mathbf{2}}$ : Here elements of $\mathcal{G}_{-}^{2 *}$ of the form $L_{+}=\xi u_{-1}+u_{0}+\xi^{-1} u_{1}+\xi^{-2} u_{2}$, span an invariant subspace under $\delta_{R_{2}} L_{+}=a d_{R_{2}}^{*}(X) L_{+}$, i.e. they form a $R_{2}$-orbit of finite functional dimension 4 . Defining the complete Lax operator $L^{(2)}=L_{+}+L_{-}=\xi u_{-1}+u_{0}+\xi^{-1} u_{1}+$ $\xi^{-2} u_{2}+\sum_{i \geq 3} \xi^{-i} w_{i-3}$ we find from eq. (19) the corresponding Hamiltonian structure to be [13]:

$$
\left(\begin{array}{cc}
P^{(2)} & 0 \\
0 & \Omega^{(2)}
\end{array}\right) \text { with } P^{(2)}=\left(\begin{array}{cccc}
0 & 0 & 0 & -u_{-1} D+u_{-1}^{\prime} \\
0 & 0 & -u_{-1} D & u_{-1} D^{2}+u_{0}^{\prime} \\
0 & -D u_{-1} & 0 & D u_{1} \\
-D u_{-1}-u_{-1}^{\prime} & -D^{2} u_{-1}-u_{0}^{\prime} & u_{1} D & u_{2} D+D u_{2}
\end{array}\right)
$$

where $P^{(2)}$ and $\Omega^{(2)}(18)$ are associated to $\left\{u_{-1}, u_{0}, u_{1}, u_{2}\right\}$ and $\left\{w_{i} \mid i \geq 0\right\}$, respectively. The LP structure $P^{(2)}$ is easily recognized as a semidirect product of (centerless) Virasoro algebra generated by the spin 2 field $u_{2}$ with a subalgebra generated by the conformal fields $\left\{u_{-1}, u_{0}+\partial_{x} u_{-1}, u_{1}\right\}$ with spins $-1,0,1$, respectively. Also, the LP structure with $\Omega^{(2)}$ (18) corresponds to the centerless algebra $\mathbf{W}_{\infty}^{\geq 3}$ which is a subalgebra of $\mathbf{W}_{\infty}$ containing all generators of spin $\geq 3$.

\section{3. "Gauge" Equivalence of Modified KP Hierarchies to Ordinary KP}

\subsection{Ordinary Coadjoint Action on R-Coadjoint Orbits as Generalized Miura Transformation}

In this section we shall explicitly construct symplectic (hamiltonian) maps among the various $R$-coadjoint orbits $\mathcal{O}(R): \Phi: \mathcal{O}(R) \longrightarrow \mathcal{O}(\widetilde{R})$, where $R, \widetilde{R}=R_{0}, R_{1}, R_{2}$. The term "symplectic" ("hamiltonian") means that under the map $\Phi$, the LP bracket structure on $\mathcal{O}(\widetilde{R})$ is transformed into the LP bracket structure on $\mathcal{O}(R)$ :

$$
\left\{\widetilde{F}_{1}, \widetilde{F}_{2}\right\}_{\widetilde{R}}(\Phi(L))=\left\{\widetilde{F}_{1}(\Phi(L)), \widetilde{F}_{2}(\Phi(L))\right\}_{R}
$$

where $\widetilde{F}_{1,2}$ are arbitrary functions on $\mathcal{O}(\widetilde{R})$ and we used notations $L$ and $\widetilde{L}=\Phi(L)$ for the coordinates on $\mathcal{O}(R)$ and $\mathcal{O}(\widetilde{R})$, respectively. As a consequence of (21), the infinite set of involutive integrals of motion $\left\{\widetilde{H}_{N}[\widetilde{L}]\right\}$ of the integrable system on $\mathcal{O}(\widetilde{R})$ are transformed into those of the integrable system on $\mathcal{O}(R): H_{N}[L]=\widetilde{H}_{N}[\Phi(L)]$.

To this end we observe that the ordinary coadjoint actions of the Lie algebra $\mathcal{G}=$ $\Psi \mathcal{D O}\left(S^{1}\right)$ and the group $G=\Psi \operatorname{DO}\left(S^{1}\right)$ on the dual space $\mathcal{G}^{*}=\Psi \mathcal{D} \mathcal{O}^{*}\left(S^{1}\right)$ do not commute with any of the $R$-coadjoint actions (16), i.e. $a d^{*}(\cdot)$ and $A d^{*}(\cdot)$ intertwine the orbits for different $R$-coadjoint actions. Thus, it is natural to look for the map $\Phi: \mathcal{O}(R) \longrightarrow \mathcal{O}(\widetilde{R})$ in 
the form :

$$
\widetilde{L} \equiv \Phi(L)=A d^{*}(g(L)) L
$$

where the group element $g(L) \in \Psi \mathrm{DO}\left(S^{1}\right)$ depends in general on the point $L$ in $\mathcal{O}(R) \subset$ $\Psi \mathcal{D O} \mathcal{O}^{*}\left(S^{1}\right)$.

It is in the sense of eqs.(21)-(22) that the integrable systems on the orbits $\mathcal{O}(R)$ for different $R$-matrices are called "gauge" equivalent. Also, from the point of view of eq.(21), i.e. mapping of one Poisson bracket structure of an integrable model into another one, the specific form of the $\Psi \mathrm{DO}\left(S^{1}\right)$ group coadjoint action (22), mapping $\mathcal{O}(R)$ into $\mathcal{O}(\widetilde{R})$, may be called generalized Miura transformation.

Let us note the following important property of $A d^{*}(g(L))$ in (22). It does not preserve the dual-projections $P_{-}^{*}, \widetilde{P}_{-}^{*}$ on $\mathcal{O}(R)$ and $\mathcal{O}(\widetilde{R})$, respectively : $\widetilde{P}_{+}^{*} A d^{*}(g(L)) P_{-}^{*} \neq 0$.

It is sufficient to prove "gauge" equivalence for linear functions on $\mathcal{O}(\widetilde{R})$, namely:

$$
\left.\{\langle\widetilde{L} \mid \widetilde{X}\rangle,\langle\widetilde{L} \mid \widetilde{Y}\rangle\}_{\widetilde{R}}\right|_{\widetilde{L}=A d^{*}(g(L)) L}=\left\{\left\langle A d^{*}(g(L)) L \mid \widetilde{X}\right\rangle,\left\langle A d^{*}(g(L)) L \mid \widetilde{Y}\right\rangle\right\}_{R}
$$

Applying the general formula (6) to the r.h.s. of (23) we have :

$$
\{\langle\Phi(L) \mid \widetilde{X}\rangle,\langle\Phi(L) \mid \widetilde{Y}\rangle\}_{R}=-\left\langle L \mid\left[\nabla_{L}\langle\Phi(L) \mid \widetilde{X}\rangle, \nabla_{L}\langle\Phi(L) \mid \widetilde{Y}\rangle\right]_{R}\right\rangle
$$

with

$\nabla_{L}\langle\Phi(L) \mid \widetilde{X}\rangle=\left(g^{-1}(L) \widetilde{X} g(L)\right)(\xi, x)-\int d y \operatorname{Res}_{\zeta}\left(\frac{\delta g(L)(\zeta, y)}{\delta L(\xi, x)} g^{-1}(L)(\zeta, y)[\widetilde{X}, \Phi(L)](\zeta, y)\right)$

\section{2 "Gauge" Transformation of $\mathrm{KP}_{\ell=1}$ to Ordinary $\mathrm{KP}$}

Let us first specialize eq.(22) to the case $\Phi: \mathcal{O}(R) \equiv \mathcal{O}\left(K P_{\ell=1}\right) \longrightarrow \mathcal{O}(\widetilde{R}) \equiv \mathcal{O}\left(K P_{\ell=0}\right)$, i.e. :

$$
\widetilde{L} \equiv \xi+\sum_{k=1}^{\infty} \xi^{-k} \widetilde{u}_{k}(x)=A d^{*}\left(g_{0}(L)\right)\left(\xi+u_{0}(x)+\xi^{-1} u_{1}(x)+\sum_{k=2}^{\infty} \xi^{-k} v_{k-2}(x)\right)
$$

One easily finds the "gauge" subgroup to be the Abelian group of multiplication operators :

$$
g_{0}(L)=\exp \phi_{0}(x) \quad, \quad \partial_{x} \phi_{0}(x)=u_{0}(x)
$$

by using the simple formula :

$$
e^{\phi_{0}(x)} \xi e^{-\phi_{0}(x)}=\xi-\partial_{x} \phi_{0}(x)
$$

Furthermore, from the structure of $\widetilde{L}(25)$ we find that only the $(+)$ parts of $\widetilde{X}$ and $\tilde{Y}$ contribute in (24), i.e. $\widetilde{X} \equiv \widetilde{X}_{\geq 0} \in \mathcal{G}_{+}^{\ell=0}(\sqrt{13})$. Finally, for $g(L)=g_{0}(L)$ (26), we have :

$$
\frac{\delta g_{0}(L)(\zeta, y)}{\delta L(\xi, x)} g_{0}^{-1}(L)(\zeta, y)=-\frac{1}{2} \varepsilon(x-y) \xi^{-1}
$$


Now specializing eq.(24) by taking into account (25)-(28) and the form of the $R$-commutator (15) for $\ell=1$, we obtain for $\widetilde{L} \equiv \exp \left(\phi_{0}\right) L \exp \left(-\phi_{0}\right)$ :

$$
\begin{aligned}
& \{\langle\widetilde{L} \mid \widetilde{X}\rangle,\langle\widetilde{L} \mid \tilde{Y}\rangle\}_{K P_{\ell=1}}= \\
& \quad-\langle\widetilde{L}| e^{\phi_{0}}\left(\left[\left(e^{-\phi_{0}} \widetilde{X} e^{\phi_{0}}\right)_{\geq 1},\left(e^{-\phi_{0}} \tilde{Y} e^{\phi_{0}}\right)_{\geq 1}\right]-\left[A_{1}\left(\phi_{0}, \widetilde{L}\right)(\widetilde{X}), A_{1}\left(\phi_{0}, \widetilde{L}\right)(\tilde{Y})\right]\right) e^{\left.-\phi_{0}\right\rangle}
\end{aligned}
$$

where $A_{1}\left(\phi_{0}, \widetilde{L}\right)(\widetilde{X}) \equiv\left(e^{-\phi_{0}} \widetilde{X} e^{\phi_{0}}\right)_{(0)}+\left(\partial_{x}^{-1} \operatorname{Res}_{\xi}[\widetilde{X}, \widetilde{L}]\right) \xi^{-1}$ and the subscript (0) means taking the zero-order part of the $\xi$-expansion of the corresponding symbol. Note that in any term on the r.h.s. of (29) of the form $\langle\widetilde{L} \mid \widetilde{Z}\rangle$ only the projection $\widetilde{Z}_{\geq-2}$ contributes. Using the simple identity :

$$
\begin{aligned}
e^{\phi_{0}}\left[\left(e^{-\phi_{0}} \widetilde{X} e^{\phi_{0}}\right)_{\geq 1},\left(e^{-\phi_{0}} \tilde{Y} e^{\phi_{0}}\right)_{\geq 1}\right] e^{-\phi_{0}} & =[\widetilde{X}, \tilde{Y}] \\
& -\left[\left(e^{-\phi_{0}} \widetilde{X} e^{\phi_{0}}\right)_{(0)}, \tilde{Y}\right]-\left[\widetilde{X},\left(e^{-\phi_{0}} \tilde{Y} e^{\phi_{0}}\right)_{(0)}\right]
\end{aligned}
$$

to rewrite the first commutator on the r.h.s. of (29), we easily find that the contribution of the terms in the second commutator on the r.h.s. of (29) are precisely canceled by the second and the third term on the r.h.s. of (30). Thus, (29) reduces to the form :

$$
\{\langle\widetilde{L}(L) \mid \widetilde{X}\rangle,\langle\widetilde{L}(L) \mid \widetilde{Y}\rangle\}_{K P_{\ell=1}}=-\langle\widetilde{L} \mid[\widetilde{X}, \widetilde{Y}]\rangle=\{\langle\widetilde{L} \mid \widetilde{X}\rangle,\langle\widetilde{L} \mid \widetilde{Y}\rangle\}_{K P}
$$

which establishes the "gauge" equivalence of $\mathbf{K P}_{\ell=\mathbf{1}}$ and $\mathbf{K P}$, i.e. that the generalized Miura-like transformation (25)-(26) maps the Poisson bracket structure of $\mathbf{K P}$ into that of $\mathbf{K} \mathbf{P}_{\ell=\mathbf{1}}$ and vice versa.

\section{3 "Gauge" Transformation of $\mathrm{KP}_{\ell=2}$ to Ordinary KP}

It is simpler to first establish the "gauge" equivalence between the modified $K P$ hierarchies $\mathbf{K P}_{\ell=\mathbf{2}}$ and $\mathbf{K P}_{\ell=\mathbf{1}}$. The desired result follows by combining the results of this and the previous subsections.

Specializing eq. (22) to the case $\Phi: \mathcal{O}(R) \equiv \mathcal{O}\left(K P_{\ell=2}\right) \longrightarrow \mathcal{O}(\widetilde{R}) \equiv \mathcal{O}\left(K P_{\ell=1}\right)$, we have

$$
\begin{aligned}
\widetilde{L} & \equiv \xi+\widetilde{u}_{0}(x)+\xi^{-1} \widetilde{u}_{1}(x)+\sum_{k=2}^{\infty} \xi^{-k} v_{k-2}(x) \\
& =A d^{*}\left(g_{1}(L)\right)\left(\xi u_{-1}(x)+u_{0}(x)+\xi^{-1} u_{1}(x)+\xi^{-2} u_{2}(x)+\sum_{k=3}^{\infty} \xi^{-k} w_{k-3}(x)\right)
\end{aligned}
$$

Here we find the "gauge" subgroup to be the (centerless) Virasoro group :

$$
\begin{aligned}
g_{1}(L) & =\exp \left(\phi_{1}(x) \xi\right) \\
u_{-1}\left(F_{\phi_{1}}(x)\right) & =\partial_{x} F_{\phi_{1}}(x) \quad \text { with } \quad F_{\phi_{1}}(x) \equiv\left(e^{\phi_{1}(x) \partial_{x}} x\right)
\end{aligned}
$$


In (34) $F_{\phi_{1}}(x)$ denotes the global group Virasoro diffeomorphism generated by the Virasoro algebra element $\phi_{1}(x) \xi \simeq \phi_{1}(x) \partial_{x}$. Note that all exponents involving symbols are operator ones. To obtain (32) one uses the simple formulas :

$$
e^{\phi_{1}(x) \xi} \xi e^{-\phi_{1}(x) \xi}=\frac{1}{\partial_{x} F_{\phi_{1}}(x)} \xi \quad, \quad e^{\phi_{1}(x) \xi} u(x) e^{-\phi_{1}(x) \xi}=u\left(F_{\phi_{1}}(x)\right)
$$

In the present case the analog of (28) reads :

$$
\frac{\delta g_{1}(L)(\zeta, y)}{\delta L(\xi, x)} g_{1}^{-1}(L)(\zeta, y)=-\frac{1}{2} \varepsilon\left(x-F_{\phi_{1}}(y)\right) \frac{1}{u_{-1}^{2}(x)} \xi^{-2} \zeta
$$

Specializing formula (24) yields for $\widetilde{L} \equiv e^{\phi_{1} \xi} L e^{-\phi_{1} \xi}$ :

$$
\begin{gathered}
\{\langle\widetilde{L} \mid \widetilde{X}\rangle,\langle\widetilde{L} \mid \widetilde{Y}\rangle\}_{K P_{\ell=2}}=-\langle\widetilde{L}| e^{\phi_{1} \xi}\left(\left[\left(e^{-\phi_{1} \xi} \widetilde{X} e^{\phi_{1} \xi}\right)_{\geq 2},\left(e^{-\phi_{1} \xi} \tilde{Y} e^{\phi_{1} \xi}\right)_{\geq 2}\right]\right. \\
\left.\left.-\left[\left(A_{2}\left(\phi_{1}, \widetilde{L}\right)(\widetilde{X})\right)_{\leq 1},\left(A_{2}\left(\phi_{1}, \widetilde{L}\right)(\widetilde{Y})\right)_{\leq 1}\right]\right) e^{-\phi_{1} \xi}\right\rangle
\end{gathered}
$$

where $A_{2}\left(\phi_{1}, \widetilde{L}\right)(\widetilde{X})=e^{-\phi_{1} \xi}\left(\widetilde{X}+\left(\partial_{x}^{-1}[\widetilde{X}, \widetilde{L}]_{(-2)}\right) \xi^{-2}\right) e^{\phi_{1} \xi}$. Similarly as in (29), in any term of the form $\langle\widetilde{L} \mid \widetilde{Z}\rangle$ on the r.h.s. of (37) only the projection $\widetilde{Z}_{\geq-2}$ contributes. The subscript $(-2)$ means taking the coefficient in front of $\xi^{-2}$ in the corresponding symbol expansion.

Noting that $A d\left(g_{1}^{-1}(L)\right)$ preserves the splitting $\widetilde{X}=\widetilde{X}_{\geq 1}+\widetilde{X}_{\leq 0}$ corresponding to $\mathbf{K P}_{\ell=\mathbf{1}}(13)$, one can rewrite (37) as :

$$
\begin{aligned}
& \{\langle\widetilde{L}(L) \mid \widetilde{X}\rangle,\langle\widetilde{L}(L) \mid \widetilde{Y}\rangle\}_{K P_{\ell=2}}=-\left\langle\widetilde{L}(L) \mid\left[\widetilde{X}_{\geq 1}, \widetilde{Y}_{\geq 1}\right]-\left[\widetilde{X}_{\leq 0}, \widetilde{Y}_{\leq 0}\right]\right\rangle \\
& +\left\langle\widetilde{L}(L) \mid\left[\widetilde{X}+\left(\partial_{x}^{-1}[\widetilde{X}, \widetilde{L}]_{(-2)}\right) \xi^{-2}, e^{\phi_{1} \xi}\left\{\left(e^{-\phi_{1} \xi} \widetilde{Y} e^{\phi_{1} \xi}\right)_{(1)} \xi\right\} e^{-\phi_{1} \xi}\right]\right\rangle \\
& -\left\langle\widetilde{L}(L) \mid\left[\tilde{Y}+\left(\partial_{x}^{-1}[\widetilde{Y}, \widetilde{L}]_{(-2)}\right) \xi^{-2}, e^{\phi_{1} \xi}\left\{\left(e^{-\phi_{1} \xi} \widetilde{X} e^{\phi_{1} \xi}\right)_{(1)} \xi\right\} e^{-\phi_{1} \xi}\right]\right\rangle
\end{aligned}
$$

Now, accounting for the structure of $\widetilde{L}(L)$ (32), one can easily show that both terms (39) and (40) vanish separately. Thus, we are left with (38) only, i.e. :

$$
\{\langle\widetilde{L}(L) \mid \widetilde{X}\rangle,\langle\widetilde{L}(L) \mid \widetilde{Y}\rangle\}_{K P_{\ell=2}}=\{\langle\widetilde{L} \mid \widetilde{X}\rangle,\langle\widetilde{L} \mid \widetilde{Y}\rangle\}_{K P_{\ell=1}}
$$

which establishes the "gauge" equivalence of $\mathbf{K P}_{\ell=\mathbf{2}}$ and $\mathbf{K} \mathbf{P}_{\ell=\mathbf{1}}$, and due to (31), also the "gauge" equivalence of $\mathbf{K P}_{\ell=\mathbf{2}}$ to ordinary $\mathbf{K P}$.

\section{Applications. A New 4-Boson Representation of $\mathbf{W}_{1+\infty}$}

Lenard Relations: As mentioned in section 2.1 above, Lenard relations shown below eq.(4) played an important role in establishing the bi-Hamiltonian structure for the ordinary $K P$ hierarchy. Here we comment on how the "gauge" equivalence between the various $K P$ 
hierarchies carries the Lenard relations over to the modified $K P$ hierarchies. First we note that $\operatorname{Tr} \widetilde{L}^{n}=\operatorname{Tr} L^{n}$, with $L$ being a Lax operator in the modified $K P$ hierarchy (notations as in (25),(32) ), follows from the "gauge "equivalence and ensures that the Hamiltonians $H_{n}$ remain identical for all $\mathbf{K P}_{\ell}$ (upon extending $H_{n}$ as functions from orbits $\mathcal{O}(R)$ to the whole dual space $\left.\mathcal{G}^{*}\right)$. For simplicity we now discuss the case of $\mathbf{K P}_{\ell=\mathbf{1}}$ with the Lax operator $L$ related through $\widetilde{L} \equiv \exp \left(\phi_{0}\right) L \exp \left(-\phi_{0}\right)$ to the Lax $\widetilde{L}$ of usual $K P$ hierarchy. One can easily show that the ordinary Lenard relations $\left\{H_{n}, \widetilde{L}\right\}_{2}=\left\{H_{n+1}, \widetilde{L}\right\}_{1}$ translate now to the new Lenard relations :

$$
\left\{H_{n+1}, L\right\}_{1}+\left[\left\{H_{n+1}, \phi_{0}\right\}_{1}, L\right]=\left\{H_{n}, L\right\}_{2}+\left[\left\{H_{n}, \phi_{0}\right\}_{2}, L\right]
$$

Especially for the two-boson $R_{1}$-orbit $L_{+}=\xi+u_{0}+\xi^{-1} u_{1}$ we get the relations $\left\{H_{n}, u_{i}\right\}_{2}=$ $\left\{H_{n+1}, u_{i}\right\}_{1}$ with $i=0,1$ reproducing the second bracket structure found in [13, 24].

New Representations of $W$-algebras : Here we will use the symplectic "gauge" equivalence map to construct new representation of the $\mathbf{W}_{\mathbf{1}+\infty}$ algebra. Let us first recall eq. (25) and solve for coefficients of the Lax operator on the l.h.s. in terms of the coefficients given on the r.h.s. of this equation. One easily finds :

$$
\widetilde{u}_{k+1}=u_{1} P_{k}\left(u_{0}\right)+\sum_{n=2}^{k+1}\left(\begin{array}{c}
k \\
n-1
\end{array}\right) v_{n-2} P_{k+1-n}\left(u_{0}\right) \quad k \geq 0
$$

where $P_{k}\left(u_{0}\right) \equiv\left(\partial+u_{0}\right)^{k} \cdot 1$, are the so called Faá di Bruno polynomials and the fields on the r.h.s. satisfy the LP bracket structure described in (20). As a corollary of the symplectic character of the "gauge" transformation we conclude that $\widetilde{u}_{k+1}$ (43) satisfy the Poissonbracket $\mathbf{W}_{\mathbf{1}+\infty}$ algebra described by the form $\Omega^{(0)}$ from (18) (note that the index labeling $\widetilde{u}_{k+1}$ is precisely equal to its conformal spin). Specifically, putting in (43) all $v_{i}$ to zero we recover the two-boson representation $\widetilde{u}_{k+1}=u_{1} P_{k}\left(u_{0}\right)$ of $\mathbf{W}_{\mathbf{1}+\infty}$ algebra described in 13, 24] (see also 250 for another related two-boson representation). The semiclassical limit is simply obtained by taking $P_{k}\left(u_{0}\right) \rightarrow u_{0}^{k}$ in (43) and yields the generators of $\mathbf{w}_{\mathbf{1}+\infty}$ algebra.

Similar considerations applied to $\mathbf{K P}_{\ell=\mathbf{2}}$ result in a new non-standard 4-boson representation of $\mathbf{W}_{\mathbf{1}+\infty}$ algebra. Indeed, performing a "gauge" transformation consisting of a composition of (25) and (32) on the 4-boson $R_{2}$-orbit $L_{+}=\xi u_{-1}+u_{0}+\xi^{-1} u_{1}+\xi^{-2} u_{2}$ with $\phi_{1}$ as in (34) and $\partial_{x} \phi_{0}(x)=\left(u_{0}+\partial u_{-1}\right)\left(F_{\phi_{1}}(x)\right)$, we obtain the following $\mathbf{K P} \widetilde{L}$ operator :

$$
\begin{gathered}
\widetilde{L}=e^{\phi_{0}} e^{\phi_{1} \xi}\left(\xi u_{-1}+u_{0}+\xi^{-1} u_{1}+\xi^{-2} u_{2}\right) e^{-\phi_{1} \xi} e^{-\phi_{0}}=\xi+\sum_{k \geq 1}^{\infty} \xi^{-k} U_{k} \\
U_{k+1} \equiv \hat{u}_{1} P_{k}\left(\hat{u}_{0}\right)+\hat{u}_{2} Q_{k-1}\left(\hat{u}_{0}, \hat{v}_{0}\right)
\end{gathered}
$$

using notations :

$$
\begin{array}{r}
\hat{u}_{0}(x) \equiv\left(u_{0}+\partial u_{-1}\right)\left(F_{\phi_{1}}(x)\right) \quad, \quad \hat{v}_{0}(x) \equiv \partial_{x} \ln \left(\partial_{x} F_{\phi_{1}}\right) \\
\hat{u}_{1}(x) \equiv \partial_{x} F_{\phi_{1}} u_{1}\left(F_{\phi_{1}}(x)\right) \quad, \quad \hat{u}_{2}(x) \equiv\left(\partial_{x} F_{\phi_{1}}\right)^{2} u_{2}\left(F_{\phi_{1}}(x)\right) \\
Q_{k}\left(\hat{u}_{0}, \hat{v}_{0}\right) \equiv \sum_{s=0}^{k}\left(\partial_{x}+\hat{u}_{0}+\hat{v}_{0}\right)^{k-s}\left(\partial_{x}+\hat{u}_{0}\right)^{s} \cdot 1 \quad(k \geq 0)
\end{array}
$$


Again, because of the symplectic property of the "gauge" transformation (44) the fields $U_{k}$ (45) span a $\mathbf{W}_{\mathbf{1}+\infty}$ LP bracket algebra realized in terms of the four bosonic fields (46) and (47). The Poisson bracket algebra of the latter :

$$
\begin{array}{r}
\left\{\hat{u}_{1}(x), \hat{u}_{0}(y)\right\}=-\partial_{x} \delta(x-y) \\
\left\{\hat{u}_{2}(x), \hat{v}_{0}(y)\right\}=-\hat{v}_{0}(x) \partial_{x} \delta(x-y)+\partial_{x}^{2} \delta(x-y) \\
\left\{\hat{u}_{2}(x), \hat{u}_{2}(y)\right\}=-2 \hat{u}_{2}(x) \partial_{x} \delta(x-y)-\partial_{x} \hat{u}_{2} \delta(x-y)
\end{array}
$$

the rest being zero, is a direct sum of the Heisenberg algebra of $\left(\hat{u}_{0}, \hat{u}_{1}\right)$ with the conformal algebra of spin 2 and non-primary spin 1 fields $\left(\hat{u}_{2}, \hat{v}_{0}\right)$. Let us point out that the deformation of the conformal algebra (50)-(51) with $\left\{\hat{v}_{0}(x), \hat{v}_{0}(y)\right\}=-c \partial_{x} \delta(x-y)$ already appeared in the construction [24] of the 2-boson representation of the second bracket structure of $\mathbf{K P}$, while the fields $\left(\hat{u}_{0}, \hat{u}_{1}\right)$ comprise the usual two-boson content of $\mathbf{W}_{\mathbf{1}+\infty}$ representation (the first term on the r.h.s. of (43)). The above 4-boson construction brings these two Bose structures together to yield a new representation (45) of the $\mathbf{W}_{\mathbf{1 + \infty}}$ algebra.

It is an interesting problem to study the quantization of the integrable system corresponding to the 4-boson realization of $\mathbf{K P}$ (quantization of the 2-boson realization of $\mathbf{K P}$ has been already undertaken in [26]).

Acknowledgements. H.A., E.N and S.P. thank S. Solomon for hospitality at the Hebrew University of Jerusalem. Support for H.A. by U.S.-Israel BSF is gratefully acknowledged. I.V. is very indebted to A. Radul for illuminating correspondence. S.P. and E.N. are thankful to CERN Theory Division and J. Ellis for hospitality during the final stage of the present work.

\section{References}

[1] D. Gross and A. Migdal, Nucl. Phys. B64 (1990) 127; M. Douglas, Phys. Lett. 238B (1990) 176; T. Banks, M. Douglas, N. Seiberg and S. Shenker, Phys. Lett. 238B (1990) 279

[2] A. Marshakov, A. Mironov and A.Morozov, Mod. Phys. Lett. A7 (1992) 1345; S. Kharchev, A. Marshakov, A. Mironov, A. Morozov and A. Zabrodin, Phys. Lett. 275B (1992) 311; A. Marshakov, On String Field Theory at $c \leq 1$, Lebedev Institute preprint, FIAN/TD-8/92, hepth/9208022

[3] M. Kontsevich, Functional Analysis and Its Application 25 (1991) 50; Intersection Theory on the Moduli Space of Curves and the Matrix Airy Function, Max-Planck Institute preprint, MPI/91-77

[4] R. Dijkgraaf, E. Verlinde and H. Verlinde, Nucl. Phys. B348 (1991) 435; R. Dijkgraaf, G. Moore and R. Plesser, The partition Function of $2 D$ String Theory, IASSNS-HEP preprint $92 / 48$, hepth/9208031

[5] M. Fukuma, H. Kawai and R. Nakayama, Comm. Math. Phys. 143 (1992) 371

[6] L.D. Faddeev and L.A. Takhtajan, Hamiltonian Methods in the Theory of Solitons, SpringerVerlag, 1987 
[7] L.A. Dickey, Soliton Equations and Hamiltonian Systems, World Scientific, Singapore (1991)

[8] A.B. Zamolodchikov, Theor. Mat. Phys. 65 (1985) 1205; A.B. Zamolodchikov and V.A. Fateev, Nucl. Phys. B280 [FS18] (1987) 644; V.A. Fateev and S.L. Lukyanov, Int. J. Mod. Phys. A3 (1988) 507

[9] I.M. Gel'fand and L.A. Dickey, Functional Analysis and Its Application 10 (1976) 13; 11 (1977) 11

[10] C. N. Pope, L. J. Romans, X. Shen, Phys. Lett. 236B (1990) 173; Nucl. Phys. B339 (1990) 191

[11] A. O. Radul, Functional Analysis and Its Application 25 (1991) 33; Sov. Phys. JETP Lett. 50 (1989) 341; Phys. Lett. 265B (1991) 86

[12] Y. Watanabe, Letters in Math. Phys. 7 (1983) 99; Ann. di Mat. Pur. Appl. 137 (1984) 77

[13] B.A. Kupershmidt, Comm. Math. Phys. 99 (1985) 51

[14] K. Yamagishi, Phys. Lett. 254B (1991) 436; F. Yu and Y.-S. Wu, Phys. Lett. 263B (1991) 220

[15] M. Douglas, N. Seiberg and S. Shenker, Phys. Lett. 244B (1990) 381

[16] B. Kostant, London Math.Soc.Lect.Notes Ser., 34 (1979) 287; M. Adler, Invent. math. 50 (1979) 219; A.G. Reyman, M.A. Semenov-Tian-Shansky and I.B. Frenkel, J. Soviet Math. 247 (1979) 802; A.G. Reyman and M.A. Semenov-Tian-Shansky, Invent. math. 54 (1979) 81; 63 (1981) 423; W. Symes, Invent. math. 59 (1980) 13

[17] F. Treves, "Introduction to Pseudo-Differential and Fourier Integral Operators", vol.1 \& 2, Plenum, New York (1980)

[18] B.A. Kupershmidt, J. Physics A23 (1990) 871

[19] D.R. Lebedev and Yu. I. Manin, Phys. Lett. 74A (1979) 154; Yu. I. Manin, J. Soviet Math. 11 (1979) 1; see also Physica 3D (1981) 400

[20] M.A. Semenov-Tian-Shansky, Functional Analysis and Its Application 17 (1983) 259

[21] M.I. Golenishcheva-Kutuzova and A.G. Reyman, J. Soviet Math. 54 (1991) 890; A.G. Reyman, J. Soviet Math. 19 (1982) 1507

[22] O. Babelon and C.-M. Viallet, Phys. Lett. 237B (1990) 411

[23] A.P. Fordy (ed.), Soliton Theory: a Survey of Results, Manchester University Press, 1990

[24] H. Aratyn, L.A. Ferreira, J.F. Gomes and A.H. Zimerman, On Two-Current Realization of KP Hierarchy, hepth/9206096; see also hepth/9201024

[25] F. Yu and Y.-S. Wu, Phys. Rev. Lett. 68 (1992) 2996; D. A. Depireux, Mod. Phys. Lett. A7 (1992) 1825

[26] M. Freeman and P. West, On the Quantum KP Hierarchy and its Relation to the Non-linear Schroedinger Equation, King's College preprint KCL-TH-92-2, hepth/9208013 